\title{
An Advanced Measurement Method to Investigate the Dynamic Development of Sediment Infiltration in an Artificial Riverbed
}

\author{
Assem MAYAR ${ }^{1, \varpi}$, Stefan HAUN $^{1}$, Markus NOACK $^{2}$, and Silke WIEPRECHT ${ }^{1}$ \\ ${ }^{1}$ Institute for Modelling Hydraulic and Environmental Systems, University of Stuttgart, Germany \\ ${ }^{2}$ Faculty of Architecture and Civil Engineering, Karlsruhe University of Applied Science, Germany \\ $\bowtie$ assem.mayar@iws.uni-stuttgart.de
}

\begin{abstract}
The dynamic development of sediment infiltration in gravel-bed rivers is not entirely understood yet because existing methods are unable to cope with the high spatio-temporal variability of the involved processes. Therefore, high-resolution and non-intrusive measurements of governing parameters are required to unravel the interactions of the multifaceted processes involved in the clogging or colmation phenomenon. This study presents high-resolution measurements of the dynamic process of sediment infiltration and the development of sediment accumulations in an artificial riverbed under laboratory conditions using an advanced non-intrusive and undisturbed method.
\end{abstract}

Keywords: gamma-ray attenuation, dynamic sediment infiltration, sediment accumulation, clogging, colmation.

\section{INTRODUCTION}

Excessive sediment infiltration and accumulation in gravel-bed rivers, intensified by anthropogenic activities, reduce pore space, conductivity, and dissolved oxygen supply, leading to severe consequences for aquatic species (Schälchli 1992; Noack et al. 2016). Owing to the several physical and bio-geo-chemical parameters involved in colmation, existing methods are unable to cope with the complex and highly dynamic behavior of the involved processes. Therefore, this study develops an advanced laboratory method for measuring the dynamic development of infiltrating sediments in an artificial riverbed with high spatial and temporal resolution.

The artificial gravel-bed was developed from $0.040 \mathrm{~m}$ and $0.026 \mathrm{~m}$ spheres in a combined cubic and rhombohedral arrangement in a laboratory flume with $0.24 \mathrm{~m}$ width, $0.30 \mathrm{~m}$ height, $8.0 \mathrm{~m}$ length, and $1.35 \%$ slope. The spheres were situated in 16 blocks with fixation from the 
top. Each block consisted of six $0.04 \mathrm{~m}$ spheres in lateral and horizontal directions and four $0.04 \mathrm{~m}$ spheres on the vertical axis leading to a total length of $3.84 \mathrm{~m}$. The $0.026 \mathrm{~m}$ spheres were glued in the concave of $0.04 \mathrm{~m}$ spheres in the cubic set up at the middle layer of the bed.

Two sediment mixtures (Fine $=1.0-1.8 \mathrm{~mm}$, Coarse $=2.0-3.5 \mathrm{~mm}$ ), with two supply rates (1.4 and $3.7 \mathrm{~kg} / \mathrm{min}$ ) and a total mass of $20 \mathrm{~kg}$, are supplied to the flume for observing the dynamic development of the sediment infiltration and accumulation processes.

The gamma-ray attenuation (GRA) method (Mayar et al. 2020) is used for non-intrusive and undisturbed continuous measurement of infiltration masses at a specific position (one-point measurement with a $15 \mathrm{~mm}$ diameter) during the entire experiments (temporal resolution of 60 seconds) and for measurements of the vertical infiltration profiles in two different intervals: (i) at the end of the sediment supply (T1), and (ii) 28 minutes after the start of the experiment (T2) with a $7 \mathrm{~mm}$ spatial resolution.

\section{RESULTS}

The results showed that the infiltrated sediment masses strongly depend on the infiltrated particle sizes. According to the vertical profiles measurement results and visual observations, the infiltrated fine sediments started filling from the bottom, while the infiltrating coarse sediment mixture resulted in a clogging layer, and subsequently, less coarse sediments penetrated to the bottom. The second time-step vertical profile measurements show that dynamic changes mostly occur in the top section due to washing of the sediments by the flow. The one-point dynamic measurement indicates higher infiltrated sediment thickness for the fine sediment mixture compared to coarse particles as a result of the clogging in the upper section. Further, the one-point measurement of the dynamic development of sediment accumulation shows that a higher supply rate leads to an earlier start of the infiltration and a more rapid filling than the lower supply rate.

\section{CONCLUSIONS}

The results proved that the GRA method is capable of non-intrusive and undisturbed measurements of sediment infiltration's dynamic development with a high spatial and temporal resolution. Within future studies, the experimental setup can be adapted (e.g., design to more naturelike conditions) to further unravel parameters involved in the highly dynamic process of sediment infiltration. Moreover, the GRA method can be coupled with other advanced flow measurement devices for an in-depth investigation of the riverbed clogging phenomenon.

Acknowledgments. The first author is funded by a scholarship from DAAD.

References

Mayar, M.A., G. Schmid, S. Wieprecht, and M. Noack (2020), Proof-of-concept for nonintrusive and undisturbed measurement of sediment infiltration masses using gamma-ray attenuation, $J$. Hydraul. Eng. 146, 5, 04020032, DOI: 10.1061/(ASCE)HY.1943-7900.0001734.

Noack, M., J. Ortlepp, and S. Wieprecht (2016), An approach to simulate interstitial habitat conditions during the incubation phase of gravel-spawning fish, River Res. Appl. 33, 2, 192-201, DOI: 10.1002/rra.3012.

Schälchli, U. (1992), The clogging of coarse gravel river beds by fine sediment, Hydrobiologia 235, 1, 189-197, DOI: 10.1007/BF00026211.

Received 22 March 2021

Accepted 12 April 2021 